\title{
A theoretical model for exciton binding energies in rectangular and parabolic spherical finite quantum dots
}

\author{
A. Taqi", J. Diouri \\ Faculté des sciences. Département de Physique, Université Abdelmalek Essaadi, \\ BP 2121, Tétouan, Morocco \\ *E-mail: abtaqi@yahoo.fr
}

\begin{abstract}
Using the variational method in real space and the effective-mass theory, we present quite an advanced semi-analytic approach susceptible for calculating the binding energy $E_{B}$ of Wannier excitons in semiconductor quantum dot structures with rectangular and parabolic shapes of the confining potential in the so-called strong-confinement regime. Illustration is given for $\mathrm{CdS}, \mathrm{ZnSe}, \mathrm{CdSe}, \mathrm{GaAs}$ structures of crystallites for both rectangular and parabolic quantum dots, and it displays a very good agreement between the experimental and theoretical results reported in literature.
\end{abstract}

Keywords: exciton, binding energy, rectangular quantum dot, parabolic quantum dot.

Manuscript received 07.04.12; revised version received 19.09.12; accepted for publication 17.10.12; published online 12.12.12.

\section{Introduction}

Recently, excitons in quantum dots have attracted more and more interest and have become the centre of attention of many experimental and theoreticals studies [1], because their original properties allow many interesting applications, namely: producing artificial atoms and molecules, single-electron transistors, and quantum dot lasers (see [2] and references cited therein). In theory, as direct solving the Hamiltonian is rather complicated and practically impossible, several attempts have been made to solve specific related problems. To determine the binding energy $E_{B}$, the variational method is commonly used with different formulations depending on the choice of a trial wave function. On the other hand, progress in experimental techniques has shown that the confinement in GaAs/GaAlAs quantum dots is approximately parabolic [3].

With regard to these important developments, we started looking for a simplified formulation, making possible a rapid and rather precise determination of exciton properties for rectangular and parabolic confining potential. Hence, we began with the usual approximation, e.g. the effective mass one, which enabled us to establish, in the framework of the variational method, general formulae for calculating the expected values of the exciton binding energy in terms of the characteristic parameters of the structure: dot radius $R_{0}$, effective masses $m_{e}$ and $m_{h}$ as well as potential profiles $V_{e}\left(r_{e}\right)$ and $V_{h}\left(r_{h}\right)$ for electron and hole forming the exciton. To illustrate them, the formulae were applied to $\mathrm{CdS}, \mathrm{CdSe}, \mathrm{ZnSe} / \mathrm{ZnS}$ and GaAs structures for rectangular and parabolic quantum dots (RQD and PQD) shapes and gave very good results.

\section{Theoretical model}

\section{1) Basic equations}

Let us consider a heterostructure consisting of a single quantum dot of type I. The confining potential is of rectangular and parabolic shapes, say $V_{e}\left(r_{e}\right)$ for electrons and $V_{h}\left(r_{h}\right)$ for holes. Then, the Hamiltonian of one electron-hole pair in the effective mass approximation [4] is as follows:

$$
H_{e x}\left(r_{e}, r_{h}\right)=H_{e}\left(r_{e}\right)+H_{h}\left(r_{h}\right)+V_{c}\left(r_{e}, r_{h}\right),
$$

where $H_{e}\left(r_{e}\right)$ and $H_{h}\left(r_{h}\right)$ are the single-particle contributions for the electron and hole, respectively, $V_{c}\left(r_{e}, r_{h}\right)=-\frac{e^{2}}{\varepsilon\left|\vec{r}_{e}-\vec{r}_{h}\right|}$ is the electron-hole Coulomb interaction $\left(\vec{r}_{e} \neq \vec{r}_{h}\right)$, and $\varepsilon$ stands for the dielectric 
constant. The single-particle Hamiltonian $H_{i}\left(r_{i}\right)$ $(i=e, h)$ is defined as:

$$
H_{i}\left(r_{i}\right)=-\nabla_{i} \frac{\hbar^{2}}{2 m_{i}\left(r_{i}\right)} \nabla_{i}+V_{i}\left(r_{i}\right),
$$

where the first term is the kinetic energy for a particle with the effective mass $m_{i}$, and $V_{i}\left(r_{i}\right)$ corresponds to the confining potential.

The problem consists in finding the eigenfunction $\Psi\left(\vec{r}_{e}, \vec{r}_{h}\right)$ and the eigenvalue $E$ of $H_{e x}$ for the ground state.

The binding energy $E_{B}$ is then related to $E$ by:

$E_{B}=E_{e}+E_{h}-E$,

where $E_{e}$ and $E_{h}$ are solutions of the one-particle problem with the ground state $\psi_{i}(i=e, h)$ :

$H_{i} \psi_{i}=E_{i} \psi_{i}$.

The general method of solution is to apply the variational principle with a trial wave function of the following form:

$\psi_{\lambda}=\psi_{e} \psi_{h} \phi_{\lambda}$.

In the strong confinement regime, the confinement effect dominates; the well dissociates the electron-hole pair, and the spatial correlation between the electron and hole is little. Then, we can choose the ansatz $\phi_{\lambda}$ as: $\phi_{\lambda}=\exp \left(-\frac{\left|r_{e}-r_{h}\right|}{\lambda}\right), \lambda$ is the variational parameter.

\section{2) Solution}

The eigenvalue $E$ of $H_{e x}$ for the ground state follows simply as the expectation value:

$E=\frac{\left\langle\psi_{\lambda}\left|H_{e x}\right| \psi_{\lambda}\right\rangle}{\left\langle\psi_{\lambda} \mid \psi_{\lambda}\right\rangle}$

$$
\text { Letting } D=\left\langle\psi_{\lambda} \mid \psi_{\lambda}\right\rangle \text {, }
$$

the numerator $N$ of Eq. (6) is given by

$N=\left\langle\psi\left|H_{e}\right| \psi\right\rangle+\left\langle\psi\left|H_{h}\right| \psi\right\rangle+\left\langle\psi\left|V_{c}\right| \psi\right\rangle=A_{e}+A_{h}+C_{e h}$,

where $A_{e, h}$ and $C_{e h}$ can be written as:

$$
\begin{aligned}
& A_{e, h}=E_{e, h} D+\frac{(4 \pi)^{2} \hbar^{2}}{2 m_{e, h}} \iint \psi_{e}^{2}\left(r_{e}\right) \psi_{h}^{2}\left(r_{h}\right)\left|\frac{\partial \phi_{\lambda}}{\partial r_{e, h}}\right|^{2} r_{e}^{2} r_{h}^{2} d r_{e} d r_{h}= \\
& E_{e, h} D+\frac{\hbar^{2}}{2 m_{e, h} \lambda^{2}} D \\
& C_{e h}=\left\langle\psi\left|V_{c}\right| \psi\right\rangle= \\
& (4 \pi)^{2} \iint \psi_{e}^{2}\left(r_{e}\right) \psi_{h}^{2}\left(r_{h}\right) V_{c}\left(r_{e}, r_{h}\right) e^{-2 \frac{\left|r_{e}-r_{h}\right|}{\lambda}} r_{e}^{2} r_{h}^{2} d r_{e} d r_{h} .
\end{aligned}
$$

In spherical harmonics, the term of electron-hole interaction can be expressed as (see for example, Marin et al. [5]): $\frac{1}{\left|\vec{r}_{e}-\vec{r}_{h}\right|}=4 \pi \sum_{l=0}^{\infty} \sum_{m=-l}^{l} \frac{1}{(2 l+1)} \frac{r_{<}^{l}}{r_{>}^{l+1}} Y_{l}^{m^{*}}\left(\theta_{e}, \phi_{e}\right) Y_{l}^{m}\left(\theta_{h}, \phi_{h}\right)$, where $r_{<}\left(r_{>}\right)$is the smaller (greater) of $r_{e}$ and $r_{h}$.

Then,

$C_{e h}=-\frac{4 \pi e^{2}}{\varepsilon} \sum_{l=0}^{\infty} \sum_{m=-l}^{l} \frac{1}{(2 l+1)} \times$

$\times \int_{\Omega_{i}} \frac{r_{<}^{l}}{r_{>}^{l+1}}\left|\psi_{e}\left(r_{e}\right)\right|^{2}\left|\psi_{h}\left(r_{h}\right)\right|^{2} e^{-\frac{2\left|r_{e}-r_{h}\right|}{\lambda}} \times$

$\times Y_{l}^{m^{*}}\left(\theta_{e}, \phi_{e}\right) Y_{l}^{m}\left(\theta_{h}, \phi_{h}\right) r_{e}^{2} r_{h}^{2} d \Omega_{e} d \Omega_{h} d r_{e} d r_{h}$,

where $d \Omega_{e}\left(d \Omega_{h}\right)$ denotes the solid angle for the electron (hole).

As $\psi_{e}\left(\right.$ or $\left.\psi_{h}\right)$ does not depend on the angles $\left(\theta_{e}, \phi_{e}\right) \quad\left(\right.$ or $\left.\left(\theta_{h}, \phi_{h}\right)\right)$,

$\int Y_{l}^{m^{*}}\left(\theta_{e}, \phi_{e}\right) d \Omega_{e}=$

$=\sqrt{4 \pi} \int Y_{l}^{m^{*}}\left(\theta_{e}, \phi_{e}\right) Y_{0}^{0}\left(\theta_{e}, \phi_{e}\right) d \Omega_{e}=\sqrt{4 \pi} \delta_{l 0} \delta_{m 0}$

and

$\int Y_{l}^{m^{*}}\left(\theta_{h}, \phi_{h}\right) d \Omega_{h}=$ $=\sqrt{4 \pi} \int Y_{l}^{m^{*}}\left(\theta_{h}, \phi_{h}\right) Y_{0}^{0}\left(\theta_{h}, \phi_{h}\right) d \Omega_{h}=\sqrt{4 \pi} \delta_{l 0} \delta_{m 0}$.

Thus, for the $1 s$ state:

$C_{e h}=-2(4 \pi)^{2} a^{*} R \iint \frac{1}{r_{>}} \psi_{e}^{2}\left(r_{e}\right) \psi_{h}^{2}\left(r_{h}\right) e^{-2 \frac{\left|r_{e}-r_{h}\right|}{\lambda}} r_{e}^{2} r_{h}^{2} d r_{e} d r_{h}$,

where $a^{*}$ and $R$ are Bohr radius $\frac{\varepsilon \hbar^{2}}{e^{2} \mu}$ and binding energy $\frac{\hbar^{2}}{2 \mu\left(a^{*}\right)^{2}}$ of the bulk exciton, respectively.

The binding energy is obtained by maximising the expectation value

$E_{B}(\lambda)=E_{e}+E_{h}-\frac{\left\langle\psi_{\lambda}\left|H_{e x}\right| \psi_{\lambda}\right\rangle}{\left\langle\psi_{\lambda} \mid \psi_{\lambda}\right\rangle}$

with respect to $\lambda$.

Then, $E_{B}(\lambda)$ can be simply written in the form:

$E_{B}(\lambda)=R\left[2 a^{*} \frac{C}{I_{0}}-\left(\frac{a^{*}}{\lambda}\right)^{2}\right]$

with

$$
\begin{aligned}
& C=\int_{0}^{+\infty} f_{e}\left(r_{e}\right)^{2} d r_{e}\left[\int_{0}^{r_{e}} \frac{1}{r_{e}} f_{h}\left(r_{h}\right)^{2} e^{-2 \frac{r_{e}-r_{h}}{\lambda}} d r_{h}+\right. \\
& \left.+\int_{r_{e}}^{+\infty} \frac{1}{r_{h}} f_{h}\left(r_{h}\right)^{2} e^{2 \frac{r_{e}-r_{h}}{\lambda}} d r_{h}\right],
\end{aligned}
$$


$I_{0}=\int_{0}^{+\infty} e^{-2 \frac{x}{\lambda}} d x \int_{0}^{+\infty}\left[f_{e}(r+x)^{2} f_{h}(r)^{2}+f_{e}(r)^{2} f_{h}(r+x)^{2}\right] d r$,

where $f_{i}\left(r_{i}\right)=r_{i} \psi_{i}\left(r_{i}\right)$ and $\mu=m_{e} m_{h} /\left(m_{e}+m_{h}\right)$ is the reduced mass of the exciton.

\section{Applications}

For illustration and with the aim of testing the validity of this model, we have calculated the exciton ground state energy in rectangular and parabolic quantum dots and compared the results with available existing data [5-7]. The agreement was very good.

\section{1) Rectangular quantum dot}

The confining potential that we assume as a spherical quantum well-like potential defined by $V_{i}\left(r_{i}\right)=\theta\left(r_{i}-R_{0}\right) V_{i}$, where $\theta$ is the step function and $V_{i}$ - barrier height.

The dependence of $m_{i}$ on $r_{i}$ arises from the fact that the particles have different effective masses depending on their location, inside or outside the dot. Then, the one-particle problem was solved by computing the solution of the following implicit eigenvalue equation for the spherical symmetry quantum dot energies [4]

$k^{i n} R_{0} \operatorname{cotg}\left(k^{i n} R_{0}\right)=1-\frac{m^{\text {in }}}{m^{\text {ou }}}\left(1+k^{o u} R_{0}\right)$.

The associated wave functions are given by:

$\psi_{i}\left(r_{i}\right)=\frac{A_{i}}{\sqrt{4 \pi}}\left[\theta\left(R_{0}-r_{i}\right) \frac{\sin \left(k_{i}^{i n} r_{i}\right)}{r_{i}}+\right.$

$\left.+B_{i} \theta\left(r_{i}-R_{0}\right) \frac{\exp \left(-k_{i}^{o u} r_{i}\right)}{r_{i}}\right]$

with $k_{i}^{i n}=\sqrt{2 m_{i}^{i n} E_{i}} ; k_{i}^{o u}=\sqrt{2 m_{i}^{o u}\left(V_{i}-E_{i}\right)}$.

The constants $A_{i}$ and $B_{i}$ are determined by normalization requirements and are equal to:

$$
\begin{aligned}
& A_{i}=\left[\frac{R_{0}}{2}-\frac{\sin \left(2 k_{i}^{\text {in }} R_{0}\right)}{4 k_{i}^{\text {in }}}+\frac{\sin ^{2}\left(k_{i}^{\text {in }} R_{0}\right)}{2 k_{i}^{\text {ou }}}\right]^{-\frac{1}{2}} \text { and } \\
& B_{i}=\exp \left(k_{i}^{\text {ou }} R_{0}\right) \sin \left(k_{i}^{\text {in }} R_{0}\right),
\end{aligned}
$$

where Rydberg units are used $\left(m_{0}=\hbar=1\right)$.

To check the accuracy of our model in spherical rectangular quantum dots, we compare our results plotted in Fig. 1 and in Table with those of refs. [5] and [6], respectively.

In Fig. 1, we display variation of the exciton ground state energy in CdS crystallites as a function of the dot radius $R_{0}$. The dashed lines represent theoretical prediction made by [5] and based on the effective-mass approximation model in the single-band scheme and the variational method, the higher curve corresponds to $V_{e}=V_{h}=2.5 \mathrm{eV}$ and $\frac{m_{o u}}{m_{i n}}=1$ and the lower one to $0.475 \mathrm{eV}$ and $\frac{m_{o u}}{m_{i n}}=1$. The solid lines represent our theoretical model, the higher curve corresponds to $V_{e}=V_{h}=2.5 \mathrm{eV}$ and $\frac{m_{o u}}{m_{i n}}=1$, while the lower one - to $0.475 \mathrm{eV}$ and $\frac{m_{o u}}{m_{i n}}=1$. In both cases, the results were evaluated for CdS material parameters $m_{e}=0.18 m_{0}$, $m_{h}=0.53 m_{0}, \frac{m_{o u}}{m_{i n}}=1$ and $\varepsilon=5.5, m_{0}-$ free-electron mass.

From the analysis of this figure, we conclude the following.

- The results obtained using our method are found to be slightly higher and closer to the experimental values reported in ref. [5] and represented in Fig. 1 by the symbols - circles and triangles.

- A decrease of dot radius is accompanied by an increase of overlap integral of the electron and hole wave functions; which causes an increase in the exciton ground state energy.

- $\quad$ The exciton ground state energy is found to reduce with decreasing the barrier height. This is due to increased penetration of the wave function into the barrier with a resulting lower Coulomb attraction.

In Table, we present our results for the exciton binding energy $E_{B}$ in $\mathrm{ZnSe} / \mathrm{ZnS}$ quantum dot to compare them with the theoretical results of Jia-Lin Zhu et al. [6]. In this work, the authors used the variational method with introduction of effective electron potentials. We used the same parameters therein $\left(m_{e}=0.16 m_{0}\right.$, $m_{h}=0.61 m_{0}, V_{e}=3279 \mathrm{meV}, \varepsilon=8.7$ for $\mathrm{ZnSe}$ and $m_{e}=$ $0.27 m_{0}, m_{h}=0.96 m_{0}, V_{e}=860 \mathrm{meV}$ for $\mathrm{ZnS}$ ). The agreement between this method and our results is quite good.

Table. Binding energy $E_{B}$ of excitons in $\mathrm{ZnSe} / \mathrm{ZnS}$ quantum dots as a function of the dot radius $R_{0}$ with $V_{e}=3279 \mathrm{meV}$ and $V_{h}=860 \mathrm{meV}$.

\begin{tabular}{|c|c|c|}
\hline \multirow{2}{*}{$R_{0}(\AA)$} & \multicolumn{2}{|c|}{ Binding energy $E_{B}(\mathrm{meV})$} \\
\cline { 2 - 3 } & our results & ref. [6] \\
\hline 22 & 115.665 & 114.7 \\
\hline 25 & 103.565 & 103.9 \\
\hline 34 & 78.821 & 81.21 \\
\hline 42 & 65.016 & 66.89 \\
\hline 56 & 49.762 & 51.99 \\
\hline
\end{tabular}




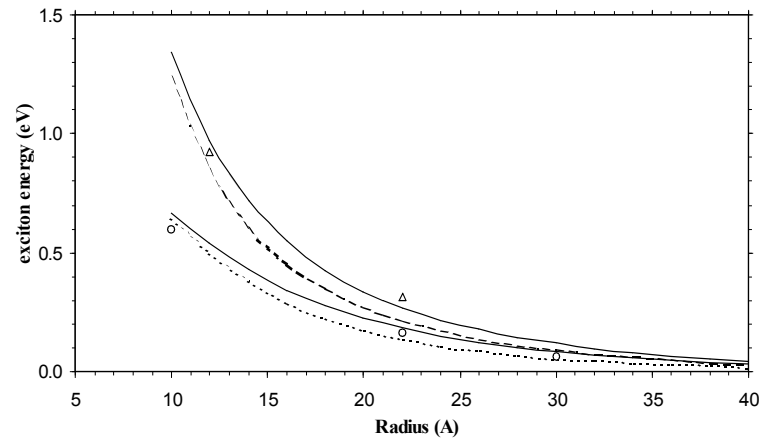

Fig. 1. Exciton ground state energy in CdS crystallites as a function of the dot radius $R_{0}$ : [5] (dots); this work (full curves).

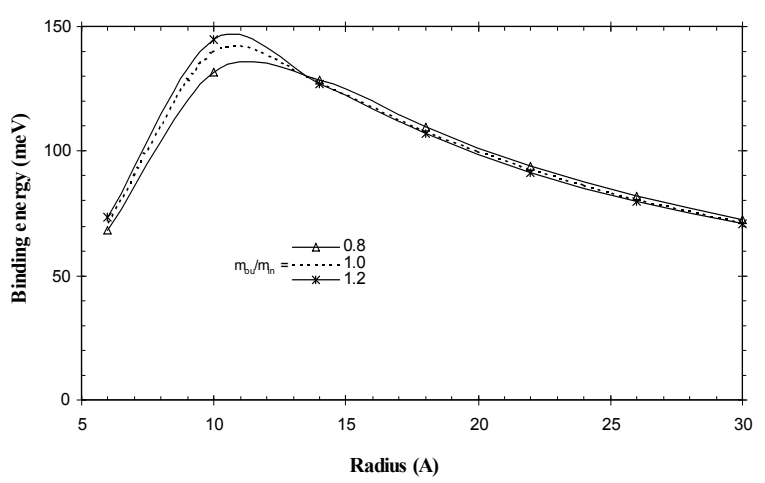

Fig. 2. Exciton binding energy in CdSe quantum dots as a function of the radius $R_{0}$ for three different values of the effective-mass ratio inside and outside the cristallite.

Fig. 2 illustrates behaviour of the ground state exciton binding energy in CdSe quantum dots as a function of the radius $R_{0}$ for three values of the effectivemass ratio $\frac{m_{o u}}{m_{i n}}=0.8,1.0$ and 1.2. The calculations were performed with the following parameters: $m_{e}=0.13 m_{0}$, $m_{h}=0.4 m_{0}, V_{e}=V_{h}=1.3 \mathrm{eV}$ and $\varepsilon=10.6$ [5].

For these three values of the effective mass ratio $\frac{m_{o u}}{m_{i n}}$ considered in this work, we found that the exciton binding energy $E_{B}$ has a maximum at a critical dot radius: on increasing $\frac{m_{o u}}{m_{i n}}$, the critical dot radius is decreased, and the maximum binding energy is increased. For a certain value of $R_{0}$, these three values become equal. We conclude that the exterior medium in which the crystallites are embedded considerably modifies behaviour of the exciton binding energy. On the other hand, we observe that the exciton binding energy increases as the radius decreases, reaching the maximum at the dot radius $\approx 10.5 \AA$, and then diminishes to a limited value corresponding to a particular radius of

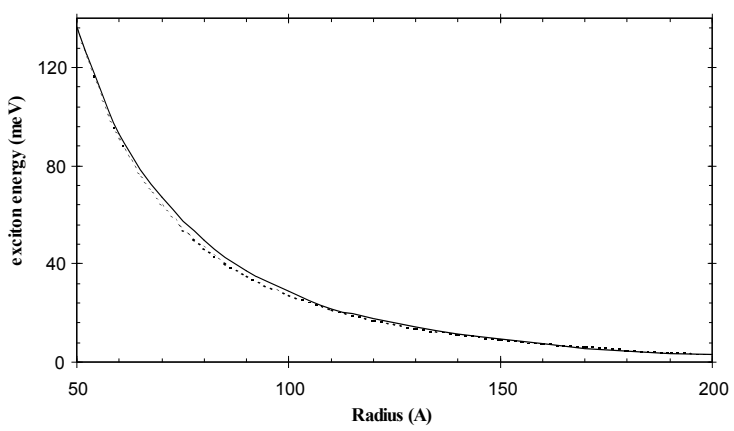

Fig. 3. Exciton ground state energy in GaAs parabolic quantum dots as a function of the dot radius $R_{0}$ : [7] (full curve); this work (dots).

the well, for which it is possible to find the free electron and hole energy level [7]. Note that for narrower dots, only confinement influences the increase of the exciton binding energy. Furthermore, as $R_{0}$ increases, the exciton binding energy approaches the energy of the unconfined two-dimensional exciton.

\section{2) Parabolic quantum dot}

In this work, we apply the model developed in Section 3 to calculate the ground state energy of excitons in a single parabolic quantum dot. The studied structure consists in a type I heterostructure with parabolic potential profiles for electrons and holes described as $V_{i}\left(r_{i}\right)=\frac{1}{2} m_{i} \Omega^{2} r_{i}^{2}[8,9]$, where $\Omega=\frac{\hbar}{\mu R_{0}^{2}}$ [8], while $R_{0}$ is the quantum dot radius, and $\mu-$ reduced mass of the exciton. Then, the ground state solutions of one-particle problem for the parabolic quantum dots are: $\psi_{i}\left(r_{i}\right) \propto \exp \left(-\alpha_{i} r_{i}^{2}\right)$ with the energy $E_{i}=\frac{3}{2} \hbar \Omega$, where $\alpha_{i}=\frac{m_{i}}{2 \hbar} \Omega$.

We have applied our model to calculate the exciton ground state energy for GaAs/GaAlAs parabolic quantum dots. By way of comparison, we have referred to the article of S. Jasiri et al. [8] and used the same parameters therein $\left(m_{e}=0.067 m_{0}, \quad m_{h}=0.377 m_{0}\right.$, $E_{g}=1520 \mathrm{meV}$, and $\varepsilon=13.1, m_{0}$ is the free-electron mass). In this work, the authors used "perturbativevariational calculations". The results obtained by this method (full curve) are very close to ours (dots) for all the range of $R_{0}$ values, as it is shown in Fig. 3.

\section{Conclusion}

A new investigation of exciton properties in rectangular and parabolic quantum dots has been performed using the advanced analytical calculations. Basic equations are derived in the framework of the commonly used approximations allowing a relatively rapid and rather precise determination of the exciton binding energy. The 
formulation applied to quantum-dot systems for RQD and PQD gives good results and may be easily extended to any given material.

\section{References}

1. M. El-Said // Sci. Technol. 9, p. 272-274 (1994).

2. G. Cantle, D. Ninno and G. Iadonosi // J. Phys.: Condens. Matter, 12, p. 9019-9036 (2000).

3. K. Brunner, U. Bockelmann, G. Abstreiter, M. Walther, G. Böhm, G. Tränkele and G. Weimann // Phys. Rev. Lett. 69, p. 3216 (1992).
4. P.G. Bolcatto and R.C. Proetto // J. Phys.: Condens. Matter, 13, p. 319-334 (2001).

5. J.L. Marin, R. Riera and S.A. Cruz // J. Phys.: Condens. Matter, 10, p. 1349-1361 (1998).

6. Zhu Jia-Lin, Zhu Shaofeng, Zhu Ziqiang, Y. Kawazoe and T. Yao // J. Phys.: Condens. Matter, 10, p. L583-L587 (1998).

7. C.A Moscoso-Moreno, R. Franco, and J. Solva-Valencia // Revista Mexicana de Fisica, 53 (3), p. 189-193 (2007).

8. S. Jasiri, and R. Bennaceur // Semicond. Sci. Technol. 9, p. 1775-1780 (1994).

9. T. Garm // J. Phys.: Condens. Matter, 8, p. 5725-5735 (1996). 